DOI: $10.15593 / 2224-9877 / 2016.2 .07$

УДК 621.921

\title{
Д.А. Кунгуров
}

Ижевский государственный технический университет им. М.Т. Калашникова, Ижевск, Россия

\section{МЕТОДИКА ОПРЕДЕЛЕНИЯ СТОЙКОСТИ ПОРЦИИ ФЕРРОМАГНИТНОГО АБРАЗИВНОГО НАПОЛНИТЕЛЯ ПРИ МАГНИТНО-АБРАЗИВНОМ ПОЛИРОВАНИИ}

\begin{abstract}
Установлено, что особенностью магнитно-абразивного полирования является непрерывное вступление в процесс резания новых режущих кромок, что относят к эффекту самозатачивания. Но со временем необратимо происходит постепенное снижение производительности полирования, связанное с разрушением зерен и насыщением порции ферромагнитного абразивного наполнителя продуктами обработки. Длительное сохранение режущей способности порции ферромагнитного абразивного наполнителя, как особого инструментального материала, используемого в технологических операциях магнитно-абразивного полирования, является важнейшим эксплуатационным показателем, влияющим на технико-экономическую эффективность производства. Установлено, что продолжительное сохранение полирующих свойств и высокой способности к микрорезанию порции ферромагнитного абразивного наполнителя зависит от многих параметров: материала абразивного компонента, макро- и микрогеометрических параметров зерен, материала и исходных параметров шероховатости поверхности, глубины дефектного слоя, схемы полирования и других условий магнитно-абразивного полирования. Определение всех этих параметров и степени их влияния на инструментальную стойкость порции ферромагнитного абразивного наполнителя в производственных условиях не представляется возможным. В связи с этим для экспериментального определения инструментальной стойкости порции ферромагнитного абразивного наполнителя предложены критерии количественной оценки инструментальной стойкости - максимально допустимый параметр шероховатости и минимальный съем материала заготовки по истечении заданного промежутка времени. Представленная в статье методика основывается на экспериментальном установлении данных количественных критериев оценки. Предложенная методика определения инструментальной стойкости может быть использована при технологической подготовке производства операций магнитно-абразивного полирования в условиях серийного производства с целью обеспечения стабильных показателей качества обработанных поверхностей при минимизации затрат на расход наполнителя. Рассчитанная по данной методике инструментальная стойкость может являться одной из сравнительных характеристик при отборе производительных наполнителей и характеризовать их абразивные свойства.
\end{abstract}

Ключевые слова: магнитно-абразивное полирование, ферромагнитный абразивный наполнитель, магнитный индуктор, инструментальная стойкость, режущие свойства, основное время, рабочий зазор, самозатачивание, зернистость наполнителя, производительность обработки, интенсивность резания, режимы обработки. 


\title{
D.A. Kungurov
}

Izhevsk State Technical University after M.T. Kalashnikov, Izhevsk, Russian Federation

\section{METHOD OF DETERMINATION OF DURABILITY OF A PORTION OF FERROMAGNETIC ABRASIVE FILLER IN MAGNETIC ABRASIVE POLISHING}

\begin{abstract}
The peculiarity of magnetic abrasive polishing is a continuous involvement of new cutting edges of the abrasive grains of the ferromagnetic filler in cutting which is related to the effect of selfsharpening. With time an irreversible degradation of polishing capacity associated with the destruction of the cutting grains and saturation of the portion of ferromagnetic abrasive filler with processing products takes place. Long-term retention of the cutting ability of the portion of ferromagnetic abrasive powder filler, as a special tool material, is the most important performance indicator influencing the technical and economic efficiency of production. It is found that long-term retention of polishing properties and high microcutting ability of the portions of ferromagnetic abrasive filler depends on many parameters: the type of abrasive material, macro- and microgeometrical parameters of grains, material and the initial parameters of roughness of the workpiece surface, the depth of the defect layer, processing scheme and other conditions of magnetic abrasive polishing. Determination of all parameters and the degree of their influence on the tool durability of a portion of ferromagnetic abrasive filler in working conditions is not possible. This is why for the experimental determination of tool durability of a portion of ferromagnetic abrasive filler proposed are criteria of quantitative assessment: the parameter of maximum allowable roughness and the minimum removal of workpiece material in the end of a specified period of time. The method described in this article was developed with the use of quantitative assessment criteria for durability. The given method presents determination of tool durability for two cases: when polishing several small workpieces by one portion of filler and when polishing a part of the surface of a large workpiece by one portion of filler. The proposed method of determination of tool durability can be used for technological preparation of production of magnetic abrasive polishing operations in the conditions of large-scale production to ensure stable parameters of the quality of processed surfaces with minimal expenditures for filler. The tool durability calculated by this method may be one of the comparative characteristics useful in selection of productive fillers.
\end{abstract}

Keywords: magnetic abrasive polishing, ferromagnetic abrasive filler, magnetic inductor, tool durability, cutting properties, most of the time, working gap, self-sharpening, filler grain, processing capacity, cutting intensity, processing condition.

В магнитно-абразивном полировании (МАП) роль режущего инструмента выполняет ферромагнитный абразивный наполнитель (далее по тексту - наполнитель), находящийся в рабочем зазоре и удерживающийся силами магнитного поля, наведенного магнитным индуктором $[1,2]$. Порция наполнителя оказывается прижатой к обрабатываемой поверхности, и при движении заготовки относительно магнитного индуктора осуществляется полирование. При этом удаляется небольшой слой материала (20-500 мкм) и формируется новый поверхностный слой с измененными физико-механическими свойствами и микрорельефом (Ra до 0,08-0,01 мкм) [3]. МАП поверхностей в зависимости от выбранных значений технологических параметров может иметь ха- 
рактеристики как способа размерной обработки связанным абразивом, так и безразмерной обработки свободным абразивом $[4,5]$.

Особенностью МАП является непрерывное вступление в процесс резания новых режущих кромок из-за перемещений зерен внутри рабочих зазоров, их поворотов и переходов, что можно называть самозатачиванием [6]. Но в процессе полирования необратимо происходит постепенное снижение производительности, связанное с разрушением зерен. Притупление режущих кромок, разрушение зерен, общее уменьшение зернистости в конечном счете вызывают возникновение дефектов в виде невыведенной исходной шероховатости [7]. Участок с таким дефектом после МАП поверхности образца из сплава Д16 при увеличении в 50 раз представлен на рис. 1.

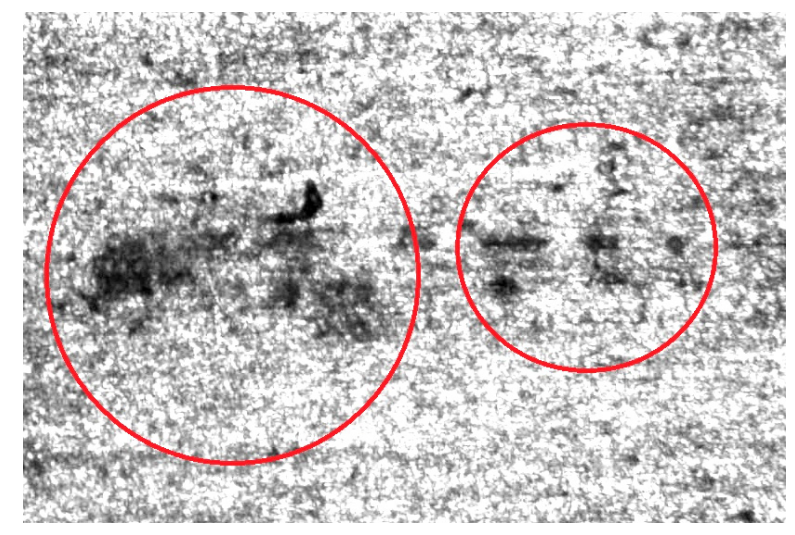

Рис. 1. Участок с невыведенной шероховатостью

Зерна наполнителя в процессе МАП контактируют с заготовкой разными участками своей поверхности с различной твердостью и геометрической формой. Очевидно, что для эффективного, длительного резания вершины зерен абразивного компонента наполнителя должны иметь малые радиусы. У композитных наполнителей можно изменять как размеры зерен, так и радиусы при вершине, тем самым управлять режущими свойствами. Увеличение зернистости наполнителя означает увеличение размеров и массы зерен, однако при большой зернистости наполнителя снижается количество зерен, одновременно контактирующих с обрабатываемой поверхностью и участвующих в резании. Высокие режущие свойства наблюдаются у наполнителей зернистостью 125-200 мкм. Режущие свойства при прочих равных условиях за- 
висят также от формы зерен, их структуры и прочности. Зерна наполнителя могут принимать изометрическую форму (близкую к сфере), осколочную удлиненную форму, а также пластинчатую форму. Изометрическая форма зерен способствует перемешиванию наполнителя в рабочих зазорах, вступлению в работу каждого зерна разными вершинами и поддерживает, таким образом, высокую производительность резания. Зерна осколочной удлиненной формы затрудняют самозатачивание порошка путем перемешивания, но имеют в пять раз большую начальную производительность, так как сначала контактируют наибольшей своей гранью, при этом период стойкости такого наполнителя небольшой. Зерна пластинчатой формы из-за низкой прочности быстро разрушаются [8-11].

Для длительного сохранения режущих свойств зерна должны иметь форму, близкую к изометрической, с несколькими острыми вершинами, торчащими в разные стороны. Такие зерна легче изменяют свое положение под действием совокупности сил в рабочем пространстве. При этом в контакт с заготовкой входят новые вершины и кромки [8]. На рис. 2 представлена предпочтительная, способствующая эффективному длительному резанию форма зерен наполнителя (изометрическая) на основе зеленого карбида кремния (зернистость 200 мкм) при увеличении в 50 раз.

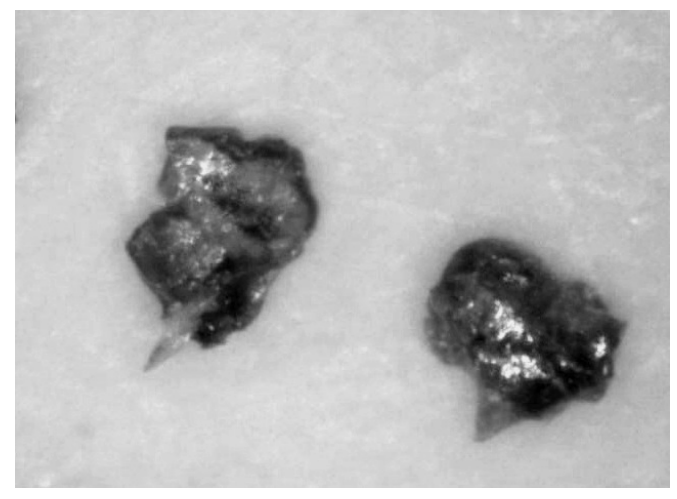

Рис. 2. Изометрическая форма зерна наполнителя

Длительное сохранение режущей способности как отдельного зерна, так и порции наполнителя характеризует инструментальную стойкость, которая является важнейшим эксплуатационным показателем, влияющим на технико-экономическую эффективность производ- 
ства. Стойкость определяет расход инструмента, затраты на подготовку и обслуживание, а также влияет на качество обработки заготовок.

Под стойкостью порции наполнителя, по аналогии с известными режущими инструментами [12], подразумевается суммарная продолжительность резания порцией наполнителя до замены из-за снижения режущих свойств ниже допустимого предела. Стойкость порции наполнителя зависит не только от магнитных и механических свойств компонентов, но и от режимов и условий МАП. В большинстве схем полирования порция наполнителя активно перемешивается и длительно сохраняет свои режущие свойства, что обусловлено эффектом самозатачивания. В других схемах перемешивание порции наполнителя в рабочем зазоре практически отсутствует и в резании участвуют одни и те же зерна, которые находятся в контакте с заготовкой и сравнительно быстро исчерпывают режущие свойства.

Причинами постепенного снижения интенсивности обработки порцией наполнителя являются: разделение магнитного и абразивного компонентов, увеличение прочностных характеристик обрабатываемой поверхности по мере удаления дефектного слоя, слабое удерживание наполнителя в рабочем зазоре, неверный подбор зернистости и (или) материалов компонентов наполнителя, насыщение массы наполнителя продуктами обработки [1]. Перечисленные факторы действуют, как правило, одновременно с преобладанием того или иного из них в конкретных условиях МАП.

Количественную оценку продолжительности резания, при которой режущие свойства порции наполнителя достигнут нижнего допустимого предела, целесообразно осуществлять с помощью параметров, характеризующих особенности МАП. Независимо от схем МАП и условий работы наполнителя (микрорезание, микровыглаживание, пластическое деформирование $[13,14])$ основой для оценки стойкости наполнителя должны быть основные закономерности процесса МАП, связанные с количеством срезанного материала с заготовки и достигнутыми параметрами шероховатости. В этом случае критериями стойкости будут служить максимально допустимые параметры шероховатости и минимальный съем материала по истечении заданного времени МАП.

Определение стойкости порции наполнителя является элементом разработки технологической операции МАП. Определить стойкость 
порции наполнителя необходимо для разработки технологической операции МАП. Когда режимы МАП уже определены, для партии заготовок и достижения стабильных результатов следует назначить основное время $t_{\mathrm{o}}$ и стойкость порции наполнителя $T$. Установление $T$ не требуется, если для полирования каждой последующей заготовки используется новая порция наполнителя, которая за время $t_{\mathrm{o}}$ не изменит режущих свойств. Методика определения $t_{\mathrm{o}}$ и $T$ при полировании относительно мелких и крупных заготовок различна.

В случае если одной порцией наполнителя последовательно полируют несколько мелких заготовок, полированием одной заготовки определяют основное время $t_{\mathrm{o}}$. Полирование при этом необходимо прерывать через короткие промежутки времени, производя измерения параметров шероховатости. По результатам проведенных измерений строится график зависимости контрольных параметров от продолжительности полирования $\tau$. График приведен на рис. 3 (для построения графика можно ограничиться контролем значений шероховатости по параметру Ra).

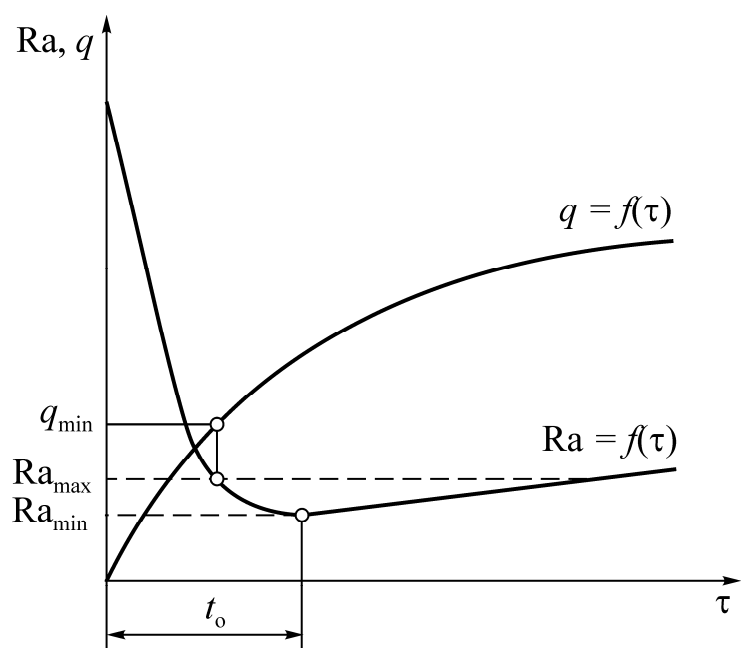

Рис. 3. График изменения контрольных параметров

Полирование при этом должно продолжаться до достижения нижней границы по параметру шероховатости Ra с минимальной заданной величиной - $\mathrm{Ra}_{\min }$. Время, необходимое для достижения $\mathrm{Ra}_{\min }$, принимается за основное время на данной операции: 


$$
\tau=t_{\mathrm{o}}
$$

После построения графика этой же порцией наполнителя последовательно полируют последующие заготовки, продолжительность обработки каждой из которых также равна $t_{0}$. Контроль $\mathrm{Ra}$ должен осуществляться только в конце обработки каждой последующей заготовки. Ввиду снижения режущих свойств используемой порции наполнителя параметр полученной шероховатости Ra будет постепенно ухудшаться. И после нескольких заготовок полученный параметр шероховатости $\mathrm{Ra}$ достигнет верхнего заданного предела $\mathrm{Ra}_{\max }$. Таким образом, время, затраченное на МАП $k$ заготовок, будет определять стойкость порции наполнителя:

$$
T=k \cdot t_{\mathrm{o}} .
$$

Если не представляется возможным установить $T$ вышеизложенным методом из-за большого объема порции наполнителя и предполагаемых значительных затрат времени, то можно найти величину стойкости другим путем. В этом случае, при определении $t_{\mathrm{o}}$ вместе с контролем параметра Ra нужно последовательно осуществлять измерение

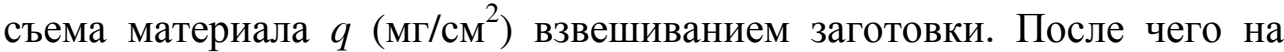
графике (см. рис. 3) построить кривую для второго контрольного параметра $q$ в зависимости от продолжительности полирования.

Величина съема материала $q$ также реагирует на изменение режущих свойств наполнителя. По эмпирическим значениям $q$, используя метод наименьших квадратов [15], по формуле (3) [16] можно определить исходную величину съема материала $q_{1}\left(\right.$ мг·мин/см$\left.{ }^{2}\right)$ и показатель $m$, который является количественной оценкой способности наполнителя сохранять свои режущие свойства в процессе МАП:

$$
q=q_{1} \cdot \tau^{m}
$$

После построения кривых $q=f(\tau)$ и $\mathrm{Ra}=f(\tau)$ согласно графику устанавливают величину минимального съема материала $q_{\min }$, необходимую для получения верхней границы по параметру шероховатости $\mathrm{Ra}$ c максимальной заданной величиной $\mathrm{Ra}_{\max }$.

Производительность МАП в любую минуту $q_{i}$ может быть определена производной от функции съема материала по времени: 


$$
\frac{\partial q}{\partial \tau}=q_{1} \cdot m \cdot \tau_{i}^{m-1}=q_{i}
$$

Необходимо также учитывать, что в случае выполнения неравенства

$$
q_{i} \cdot t_{\mathrm{o}} \leq q_{\min }
$$

полирование последующих заготовок этой порцией наполнителя не обеспечит получения шероховатости в пределах $\mathrm{Ra}_{\min }<\mathrm{Ra}<\mathrm{Ra}_{\max }$. В этом случае порция наполнителя подлежит замене, и стойкость будет определяться по следующей формуле:

$$
T=\tau_{i} \text {. }
$$

После подстановки выражений (5) и (6) в формулу (4) получим выражение для расчета стойкости порции порошка:

$$
T=\sqrt[1-m]{\frac{m \cdot q_{1} \cdot t_{\mathrm{o}}}{q_{\min }}} .
$$

В случае МАП крупных заготовок вышеизложенная методика не может быть использована, так как необходимо определить период замены порции наполнителя при полировании всей площади поверхности заготовки. Заготовка полируется с подачей магнитного индуктора вдоль поверхности, поэтому участки поверхности будут обработаны с разной интенсивностью из-за снижения режущих свойств порции наполнителя. В этой связи предлагается другая методика определения стойкости при обработке крупных заготовок. Условия и режимы МАП могут быть испытаны на контрольном образце. При этом на поверхности контрольного образца устанавливают вставные элементы, обрабатываемая поверхность которых расположена на одном уровне с поверхностью контрольного образца. Материал и шероховатость поверхности испытуемого контрольного образца должны соответствовать исходной реальной заготовке. МАП осуществляется с подачей магнитного индуктора вдоль обрабатываемой поверхности, но при значительно меньшей длине хода. Измерения производят только на вставных элементах.

Требуемой шероховатости на большой площади поверхности можно достичь за один проход магнитного индуктора с небольшой

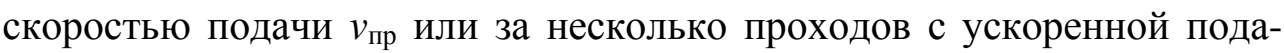


чей. В первом случае необходимо экспериментально установить на контрольном образце величину скорости подачи $v_{\text {пр }}$, при которой за один проход с одной порцией наполнителя обеспечится $\mathrm{Ra}_{\min }$. Обрабатывая этой же порцией наполнителя последовательно несколько вставных элементов, можно определить порядковый номер образца $N$, на котором за один проход магнитного индуктора обеспечится $\mathrm{Ra}_{\max }$. Длина одного прохода магнитного индуктора в эксперименте - $S$. Тогда длина поверхности заготовки, на которой одной порцией наполнителя обеспечится шероховатость в заданных пределах, определится по формуле

$$
L=N \cdot S .
$$

Отсюда стойкость порции наполнителя будет

$$
T=\frac{N \cdot S}{v_{\text {пр }}} .
$$

Во втором способе МАП (за несколько проходов с ускоренной

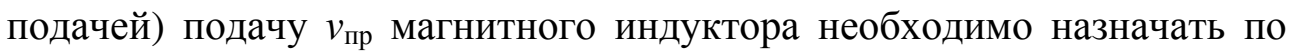
рекомендациям или на основании опыта. Экспериментально на контрольном образце получают зависимости съема материала и параметра шероховатости от количества проходов $i$ :

$$
\begin{gathered}
q=q_{1} \cdot i^{m}, \\
\mathrm{Ra}=f(i) .
\end{gathered}
$$

С помощью зависимости (11) можно определить количество проходов магнитного индуктора $i_{0}$, необходимое для получения шероховатости $\mathrm{Ra}_{\min }$. Основное время полирования $t_{\mathrm{o}}$ с учетом $i_{\mathrm{o}}$ можно определить по формуле

$$
t_{\mathrm{o}}=\frac{i_{\mathrm{o}} \cdot L}{v_{\text {пр }}} .
$$

Производительность МАП при каждом последовательном ходе магнитного индуктора $q_{i}$ может быть определена производной от функции съема материала по количеству проходов:

$$
\frac{\partial q}{\partial i}=q_{1} \cdot m \cdot i^{m-1}=q_{i} .
$$


Введем коэффициент допускаемого снижения производительности МАП порцией наполнителя $\varepsilon$, который определяется по формуле

$$
\varepsilon=\frac{q_{i}}{q_{1}}
$$

После подстановки выражения (14) в формулу (13) определим количество проходов $i$, после которых происходит снижение производительности МАП порцией наполнителя:

$$
i=\sqrt[1-m]{\frac{m}{\varepsilon}}
$$

Поскольку длина каждого прохода магнитного индуктора $S$

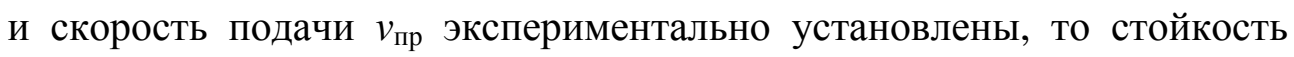
порции наполнителя будет определяться по формуле

$$
T=\frac{i \cdot S}{v_{\text {пр }}}=\sqrt[1-m]{\frac{m}{\varepsilon}} \cdot \frac{S}{v_{\text {пр }}} .
$$

Показатель $m$, устанавливаемый экспериментально во всех случаях, может служить оценочной характеристикой абразивных свойств определенного состава наполнителя. При сравнении различных составов наполнителей при полировании деталей из одного материала и с одинаковой технологической наследственностью увеличенные значения показателя $m$ будут характеризовать повышенную стойкость. Определение показателя $m$ разных составов наполнителей позволит установить состав с наибольшей стойкостью порции. Такой наполнитель может работать в более тяжелых с точки зрения нагрузок условиях.

Изложенная методика расчета инструментальной стойкости порции наполнителя может быть использована при разработке операций МАП с целью достижения стабильных показателей качества партии деталей и определения момента критического износа порции для ее замены.

\section{Список литературы}

1. Барон Ю.М. Технология абразивной обработки в магнитном поле. - Л.: Машиностроение, 1978. - 128 с.

2. Коновалов Е.Г., Шулев Г.С. Чистовая обработка деталей в магнитном поле ферромагнитными порошками. - Минск: Наука и техника, 1967. - $125 \mathrm{c}$. 
3. Отделочно-абразивные методы обработки: справ. пособие / Л.М. Кожуро, А.А. Панов, Э.Б. Пономарева, П.С. Чистосердов / под общ. ред. П.С. Чистосердова. - Минск: Выш. шк., 1983. - 287 с.

4. Хомич Н.С. Магнитно-абразивная обработка изделий: монография. - Минск: Изд-во Белорус. нац. техн. ун-та, 2006. - 218 с.

5. Прогрессивные методы абразивно-алмазной обработки / П.И. Ящерицын, Е.Э. Фельдштейн, Н.С. Хомич, В.И. Прибыльский // Материалы, технологии, инструменты. - 1997. - № 2. - С. 44-52.

6. Барон Ю.М. Магнито-абразивная и магнитная обработка изделий и режущих инструментов. - Л.: Машиностроение, 1986. - 176 с.

7. Сакулевич Ф.Ю., Минин Л.К., Олендер Л.А. Магнитно-абразивная обработка точных деталей. - Минск: Выш. шк., 1977. - 288 с.

8. Кунгуров Д.А., Мкртчян А.Ф. Исследование геометрических параметров абразивных зерен в магнитно-абразивной обработке [Электронный ресурс] // Современные проблемы науки и образования. 2014. - № 4. - URL: http://www.science-education.ru/118-14140 (дата обращения: 12.02.2016).

9. Маслов Е.Н. Теория шлифования материалов. - М.: Машиностроение, 1974. - 320 с.

10. Ящерицын П.И., Жолнерович Е.А. Шлифование металлов. Минск: Беларусь, 1970. - 462 с.

11. Martin R. Neue Erkentnisse über den Werkstoffabtragsvorgang beim Lappen // Facher. Oberflachentechn. -1972. - № 6. - S. 197-202.

12. Справочник технолога-машиностроителя: в 2 т. / под ред. А.М. Дальского, А.Г. Суслова, А.Г. Косиловой, Р.К. Мещерякова. 5-е изд., перераб. и доп. - М.: Машиностроение-1, 2001. - Т. 2 - 944 с.

13. Сакулевич Ф.Ю. Основы магнитно-абразивной обработки. Минск: Наука и техника, 1981. - 328 с.

14. Маталин А.А. Новые направления развития технологии чистовой обработки. - Киев: Техника, 1972. - 136 с.

15. Кацев П.Г. Статистические методы исследования режущего инструмента. - М.: Машиностроение, 1974. - 239 с.

16. Барон Ю.М. Физические основы работы магнитно-абразивных материалов // Магнитно-абразивные материалы и методы их испытаний. - Киев: Изд-во Ин-та проблем материаловедения АН УССР. - 1980. - С. 10-17. 


\section{References}

1. Baron Iu.M. Tekhnologiia abrazivnoi obrabotki v magnitnom pole [Technology of abrasive processing in a magnetic field]. Leningrad: Mashinostroenie, $1978.128 \mathrm{p}$.

2. Konovalov E.G., Shulev G.S. Chistovaia obrabotka detalei v magnitnom pole ferromagnitnymi poroshkami [Fair processing of details in a magnetic field ferromagnetic powders]. Minsk: Nauka i tekhnika, 1967. $125 \mathrm{p}$.

3. Kozhuro L.M., Panov A.A., Ponomareva E.B., Chistoserdov P.S. Otdelochno-abrazivnye metody obrabotki [Finishing and abrasive methods of processing]. Minsk: Vysshaia shkola, 1983. $287 \mathrm{p}$.

4. Khomich N.S. Magnitno-abrazivnaia obrabotka izdelii [Magnetic and abrasive processing of products]. Minsk: Belorusskii natsional'nyi tekhnicheskii universitet, 2006. 218 p.

5. Iashcheritsyn P.I., Fel'dshtein E.E., Khomich N.S., Pribyl'skii V.I. Progressivnye metody abrazivno-almaznoi obrabotki [Progressive methods of abrasive and diamond processing]. Materialy, tekhnologii, instrumenty, 1997, no. 2, pp. 44-52.

6. Baron Iu.M. Magnito-abrazivnaia i magnitnaia obrabotka izdelii i rezhushchikh instrumentov [Magneto-abrasive and magnetic processing of products and the cutting tools]. Leningrad: Mashinostroenie, 1986. $176 \mathrm{p}$.

7. Sakulevich F.Iu., Minin L.K., Olender L.A. Magnitno-abrazivnaia obrabotka tochnykh detalei [Magnetic and abrasive processing of exact details]. Minsk: Vysshaia shkola, 1977. 288 p.

8. Kungurov D.A., Mkrtchian A.F. Issledovanie geometricheskikh parametrov abrazivnykh zeren $\mathrm{v}$ magnitno-abrazivnoi obrabotke [Research of geometrical parameters of abrasive grains in magician-nitno-abrasive processing]. Sovremennye problemy nauki i obrazovaniia, 2014, no. 4, available at: http://www.science-education.ru/118-14140 (accessed 12 Febryuary 2016).

9. Maslov E.N. Teoriia shlifovaniia materialov [Theory of grinding of materials]. Moscow: Mashinostroenie, 1974. 320 p.

10. Iashcheritsyn P.I., Zholnerovich E.A. Shlifovanie metallov [Grinding of metals]. Minsk: Belarus', 1970. 462 p.

11. Martin R. Neue Erkentnisse über den Werkstoffabtragsvorgang beim Lappen. Facher. Oberflachentechn, 1972, no. 6, pp. 197-202. 
12. Dal'skii A.M., Suslov A.G., Kosilovoi A.G., Meshcheriakova R.K. Spravochnik tekhnologa-mashinostroitelia [Reference book of the technologist-mechanician]. Moscow: Mashinostroenie, 2001. Vol. 2. 944 p.

13. Sakulevich F.Iu. Osnovy magnitno-abrazivnoi obrabotki [Bases of magnetic and abrasive processing]. Minsk: Nauka i tekhnika, 1981. 328 p.

14. Matalin A.A. Novye napravleniia razvitiia tekhnologii chistovoi obrabotki [New directions of development of technology of fair processing]. Kiev: Tekhnika, 1972. 136 p.

15. Katsev P.G. Statisticheskie metody issledovaniia rezhushchego instrumenta [Statistical methods of research of the cutting tool]. Moscow: Mashinostroenie, 1974. 239 p.

16. Baron Iu.M. Fizicheskie osnovy raboty magnitno-abrazivnykh materialov [Physical bases of work of magnetic and abrasive materials]. Magnitno-abrazivnye materialy $i$ metody ikh ispytanii. Kiev: Institut problem materialovedeniia Akademii nauk USSR, 1980, pp. 10-17.

Получено 16.03.2016

\section{Об авторе}

Кунгуров Денис Андреевич (Ижевск, Россия) - аспирант кафедры «Автомобили и металлообрабатывающее оборудование» Ижевского государственного технического университета им. М.Т. Калашникова; e-mail: d_kungurov@mail.ru.

\section{About the author}

Denis A. Kungurov (Izhevsk, Russian Federation) - Postgraduate Student, Department of Vehicles and Metalworking Equipment, Izhevsk State Technical University after M.T. Kalashnikov; e-mail: d_kungurov@ mail.ru. 